\title{
INTEGRATED CARE Interdisciplinary research: shaping the healthcare of the future
}

\author{
Authors: Stephen W Smye ${ }^{A}$ and Alejandro F Frangi ${ }^{B}$
}

The hospitals of the future will be shaped by scientific and technical advances made across a wide range of disciplines because complex problems in healthcare cannot be addressed successfully by a single discipline. This paper considers how interdisciplinary research is being promoted and the prospects for developing stronger and deeper collaborations between medicine, health and other disciplines, drawing on case studies from mathematics, physics and engineering. The anticipated impact of greater interdisciplinarity on clinical training and the provision of care is also reviewed. While the role and training of clinicians in the provision of care will continue to evolve, they will remain leading members of a much broader and more diverse interdisciplinary team, alert to the value of deep and sustained interdisciplinary research.

KEYWORDS: research, partnerships, interdisciplinary

DOI: $10.7861 /$ fhj.2021-0025

\section{Introduction}

Medicine has a long history of drawing on other disciplines to make significant advances in clinical care. Examples of clinical advances driven by disciplines other than medicine abound, for example, many techniques and technologies which underpin modern clinical imaging originated in the physics laboratory; radiotherapy is founded on the pioneering work of the physicist and chemist, Marie Curie, and the engineer and physicist, William Roentgen. The ground-breaking work of Franklin (chemist), Crick (physicist), Watson (biologist) and Wilkins (physicist) in determining the structure of DNA is now secure in the public record. The impressive advances in microscopy, which have been central to our understanding of biology, were driven in part by advance in physics and the demands of materials scientists.

In more recent times, the value of mathematics as a framework for understanding the burgeoning data sets arising from new, clinically informative, measurements is increasingly recognised

Authors: ${ }^{A}$ professor, University of Leeds School of Medicine, Leeds, UK; ${ }^{B}$ diamond jubilee chair in computational medicine and Royal Academy of Engineering chair in emerging technologies, University of Leeds Schools of Computing and Medicine, Leeds, UK and has led to a notable migration of mathematical physicists with doctorates in areas such as string theory, cosmology and quantum field theory into medicine where their mathematical skills are being used to, for example, advance epidemiological modelling, develop novel clinical trials and understand tumour dynamics. ${ }^{1-4}$ Artificial intelligence, which has manifold applications in healthcare, originated, in part, from Turing's 1950 paper Computing machinery and intelligence and is underpinned by advances in fundamental computer science. ${ }^{5}$

The report commissioned by the Engineering and Physical Sciences Research Council and produced by an expert panel chaired by Prof Patrick Maxwell in 2014, on the importance of engineering and the physical sciences to medicine gives numerous examples of interdisciplinary research and noted that 'During the last 35 years, 11 of the Nobel prize-winners for medicine have had a background in chemistry, physics or engineering. ${ }^{6}$

Examples of interdisciplinary research are not limited to the science, technology, engineering and mathematics (STEM) subjects; social, economic and behavioural sciences play a prominent role in improving public health and clinical practice. The role of the arts and wider humanities has been the focus of several recent initiatives. ${ }^{7}$

At the time of the founding of the Royal College of Physicians in 1518 , individual physicians often embodied interdisciplinarity. The subsequent advances in medical science have led to an almost inevitable stratification of the research community and the emergence of specialty-specific clinical practice and research endeavour. While this focused approach to research has undoubtedly led to significant progress, it has also been widely recognised that the 'silo-thinking', which may be associated with a uni-disciplinary approach, can be very limiting and misses the real opportunities for advance provided by disciplines other than medicine or indeed biology. Interestingly, a citation-based analysis of interdisciplinary research over the period 2001-2010 undertaken by Van Noorden and colleagues demonstrates that interdisciplinary research is increasingly prominent, particularly in 'health' but that 'clinical medicine' is strongly uni-disciplinary in the sense that 'Clinical medicine papers rarely cite, or are cited by, other disciplines, perhaps because they deal with specialised medical practice.' (Fig 1). ${ }^{8}$ This suggests there is more to be done to promote interdisciplinary approaches.

Rylance rehearses the drivers of interdisciplinary research; complex problems are not amenable to single-discipline investigation, discoveries are more likely at the boundaries between disciplines, and single disciplines derive a general benefit 


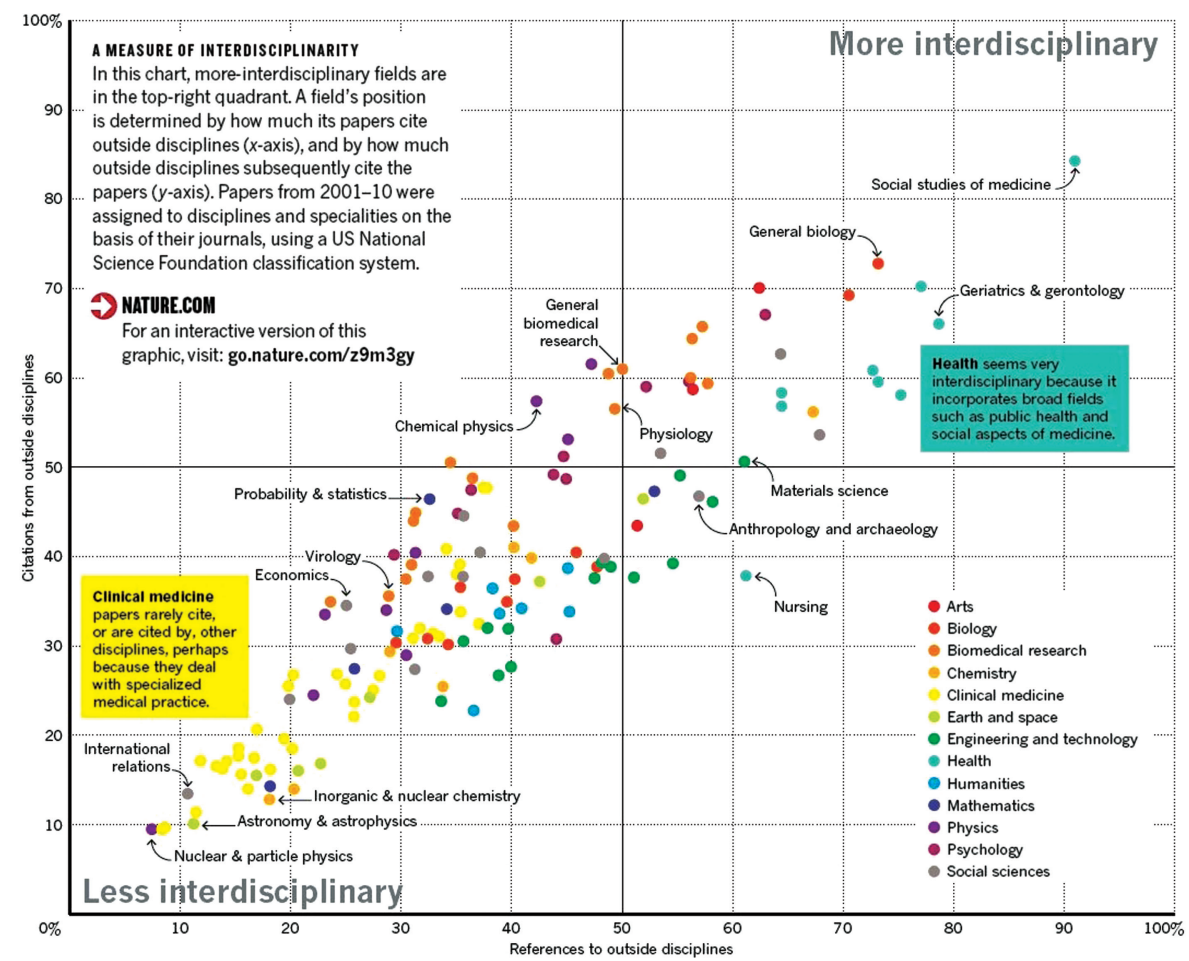

Fig 1. Measure of interdisciplinarity of fields. Reproduced with permission from Van Noorden R. Interdisciplinary research by the numbers. Nature 2015;525:306-7. from engagement with those outside their field. ${ }^{9}$ Rylance also summarises the reasons given for being reticent to embrace interdisciplinarity; such research is often regarded as inferior to that in a single discipline (with publication metrics being cited as the rationale for this) that promoting interdisciplinarity can sometimes be at the expense of the individual disciplines. ${ }^{9}$

Wu, Wang and Evans further demonstrate that science and technology can be disrupted by small teams, yet larger and often interdisciplinary teams are crucial to demonstrate impact and change practice. ${ }^{10}$ Their results also suggest the need to support the critical role small teams appear to have in paradigm shifts and challenging the frontiers of knowledge. Here, we make the case that hospitals of the future will be shaped by a deep and sustained interdisciplinary approach to healthcare research. The term 'interdisciplinary' is used in slightly different ways, often interchangeably with the terms 'cross-disciplinary', 'multidisciplinary' and 'transdisciplinary'. In this paper, we use the term 'interdisciplinary' to refer to the full spectrum of activities which span more than a single discipline. Medicine is here considered as a single discipline, and the focus of the paper is on the contributions being made to clinical care from disciplines outside medicine. The paper briefly summarises some of the key principles underpinning high-quality, interdisciplinary research and notes several recent initiatives designed to promote productive research collaborations between medicine and other disciplines. The paper then illustrates some of these principles with reference to the collaborations between medicine and the physical, engineering and mathematical sciences. The implications of interdisciplinary research for clinical training are briefly reviewed. The paper concludes with some speculation about future developments in interdisciplinary research, which may significantly impact future healthcare.

\section{Principles}

A useful compendium of recent reports on interdisciplinary research has been curated by UK Research and Innovation (UKRI). ${ }^{11}$ Principles and processes which underpin successful interdisciplinary research are increasingly recognised and are set out in detail by Brown, Deletic and Wong. ${ }^{12}$ We select three of particular importance to medicine and summarise them.

> Promote depth, so a researcher becomes 'T-shaped': someone with real credibility in their field but who can look beyond it. The implications for career development, particularly for early career researchers, are significant and require careful management if career progression is not to be delayed. ${ }^{12}$ In the UK, recent reports on 'team science', including a report from The Academy of Medical Sciences, have helpfully contributed to addressing this important aspect of interdisciplinary research. ${ }^{13}$

> Focus on a 'grand challenge': grand challenges abound in medicine and, almost by definition, addressing a grand challenge requires researchers to look beyond their field. Cancer Research UK, working with the National Cancer Institute, has been in the vanguard of this approach with global teams, comprising many disciplines, each funded on a scale that reflects the significance of the scientific question. ${ }^{14}$ The Council for Science and Technology recently advised the prime minister on creating ambitious, goal-oriented research and development programmes based on seven principles for science and innovation 'moon-shots'.15

> Nurture dialogue across traditional boundaries: interdisciplinary seminars, collocated research groups and facilities, PhD cohorts with cross-disciplinary supervisory teams and shared coffee lounges drive better mixing with other disciplines. Even in large teaching hospitals with established university partnerships, 
Fig 2. Interdisciplinary working in the NIHR Surgical MedTech Cooperative at Leeds aimed at addressing unmet needs. NIHR = National Institute for Health Research; RCTs = randomised controlled trials. Adapted from a diagram produced by Vee Mapunde, University of Leeds and Leeds Teaching Hospitals NHS Trust.

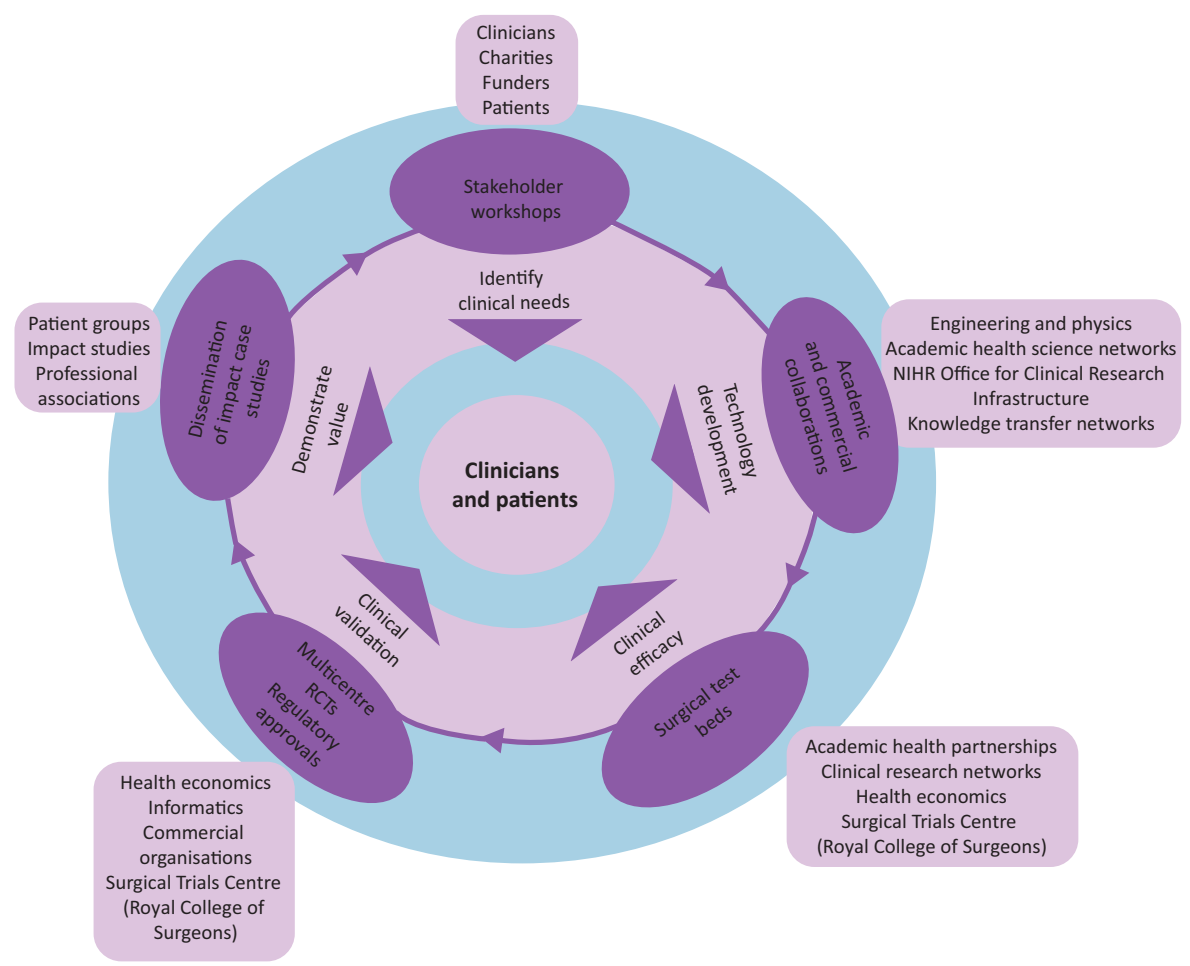

promotion of mixing between disciplines is not always easy and is often severely time limited. The issue of protecting clinician time for research, including research which involves crossdisciplinary collaborations, is widely recognised and solutions are being proposed. ${ }^{16,17}$ Interdisciplinary collaborations are often best supported by a programme of engagement planned and supported by established research platforms, including those funded by the National Institute for Health Research (NIHR); for example, in Leeds, the NIHR Surgical MedTech Cooperative drives collaborations at scale between clinicians, physicists, engineers, industry and patients aimed at addressing unmet needs (Fig 2). ${ }^{18}$ Further upstream in the research pipeline, the Bragg Centre for Advanced Materials Research has a regular programme of events which promote novel interdisciplinary approaches to key clinical topics, such as drug targeting and delivery. ${ }^{19}$

> Leading from the front: future hospitals are not defined by new technology but by a culture shift in understanding the scope of care provision, their boundaries and the speed of change in which a culture of constructive questioning is encouraged. ${ }^{20,21}$ Leaders and middle managers are key agents in this transition by providing the incentives and recognising the needed space and upskilling for a successful transition. A transition that requires the recognition that clinical research and innovation is a statutory right and duty of NHS practitioners to stay current and provide the best patient care. The NIHR is working closely with NHS England, regulators and the medical royal colleges to ensure research becomes embedded in clinical care.

National and international programmes aimed at driving interdisciplinary collaborations at scale, and reflecting these principles, are numerous.
> The UKRI-funded Physics of Life network (PoLNet3), now in its third cycle, which also includes a programme of events, funded by the Rosetrees Trust, aimed at strengthening clinical engagement with the biological physics and related communities in the basic sciences (the Physics of Medicine). ${ }^{22}$ A summer school focusing on biomedicine is planned. The Rosetrees Trust have an established history of supporting deeply-interdisciplinary research.

> UKRI funds a wide range of fellowship schemes, many of which are aimed at interdisciplinary research. ${ }^{23}$

$>$ NIHR funds fellowships that are well-suited to those looking to collaborate with other disciplines. ${ }^{24}$

> The Engineering and Physical Sciences Research Council (EPSRC) has a very strong grants programme for the healthcare technology, including funding for interdisciplinary fellowship and networks, aimed at developing new approaches to some of the grand challenges in healthcare. ${ }^{25}$

> EPSRC also funds hubs for mathematics in healthcare: the Exeter Hub for Quantitative Modelling in Healthcare, for example, includes clinicians and aims to develop new methods for managing and treating diabetes, mental health and microbial disease using predictive mathematical models. ${ }^{26}$

> Cancer Research UK's Multidisciplinary Award scheme: the scheme is co-funded by EPSRC and requires at least one investigator from the engineering or physical sciences. ${ }^{27}$

> In the USA, the National Cancer Institute's (NCI's) Physical Sciences in Oncology Initiative seeks to establish research projects that bring together cancer biologists and oncologists with scientists from physics, mathematics, chemistry and engineering to address some of the major questions and barriers in cancer research. ${ }^{28}$ 


\section{Making an impact in the clinic}

There are numerous examples of interdisciplinary research that make a difference in the clinic or have the potential to do so. The following examples illustrate how interdisciplinary groups addressing a clinical question to which the answer really matters can make significant advances. All major disease areas can furnish examples, with cancer particularly prominent and well-supported by research funding schemes which drive interdisciplinarity.

One of the most striking demonstrations of the clinical impact of interdisciplinary research in cancer is provided by the Moffitt Cancer Centre's NCI-funded programme on cancer biology and evolution (CBE) which includes a particular focus on the problem of treatment resistance and is described as:

... emerg[ing] from systematic in-house collaborations of mathematicians, evolutionary biologists, and basic and clinical cancer researchers. Although these research teams investigate cancer via traditional means, they include mathematicians and theorists who integrate multi-scalar data through quantitative models founded on evolutionary first principles.

The overall goals of CBE are to investigate and define the complex dynamics that govern the biology and therapeutic responses of cancer, and to deliver new agents and strategies to prevent and treat refractory or relapsed malignancies. ${ }^{29}$

While the role of evolutionary dynamics is well-recognised in the emergence of treatment resistance in cancer (and draw significantly on earlier work on the role of selection in ecological systems), the Moffitt centre has used these approaches to design novel drug trials. These trials are based on the observation that using the 'maximally tolerated dose' is likely to accelerate the emergence of resistance clones in the tumour. This process can be modelled mathematically, thereby enabling the trajectory of the tumour's clonal evolution to be predicted. A treatment regimen, which uses a lower dose, stopped after achieving a pre-determined tumour response and only restarted when tumour growth reaches a pre-set level, has clear theoretical advantages in slowing the rate of emergence of resistant clones and mathematical simulations suggest that 'driving tumour evolution into periodic, repeatable treatment cycles provides a path forward for multidrug adaptive therapy' (Fig 3). ${ }^{30}$ Clinical trials based on this approach are now underway, and early results are promising. ${ }^{31}$

Targeting cytotoxic drugs to tumour tissue while avoiding the toxicity in normal tissue is another major problem in cancer treatment and one benefiting from novel approaches rooted in the physics, chemistry and applications of nanomaterials, combined with imaging techniques; for example, recent collaborative work, which included researchers in the schools of medicine, chemistry and physics and astronomy at Leeds University has demonstrated that ultrasound-triggered therapeutic microbubbles enhance the efficacy of cytotoxic drugs by increasing circulation and tumour drug accumulation, while also limiting bioavailability and toxicity in normal tissues. ${ }^{32}$

Even some of the most fundamental areas of research in physics offer opportunities for clinical translation; for example, Ben Varcoe, professor of quantum information science at Leeds University, uses an error correction mechanism drawn from quantum optics to improve the signal-to-noise ratio of magneto-cardiography significantly and thereby develop a device for clinical use. ${ }^{33}$ This work has been undertaken in close collaboration with cardiologists and emergency medicine physicians.
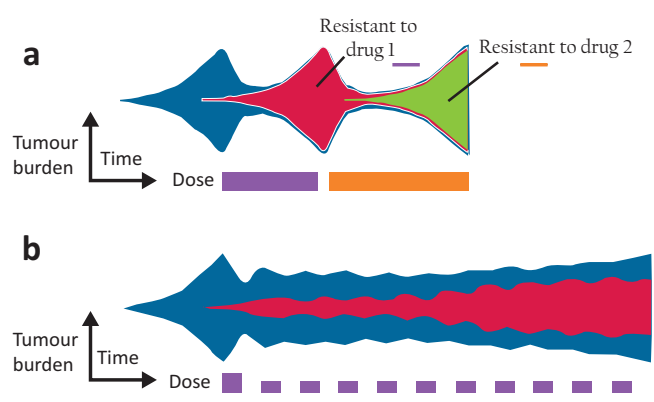

C

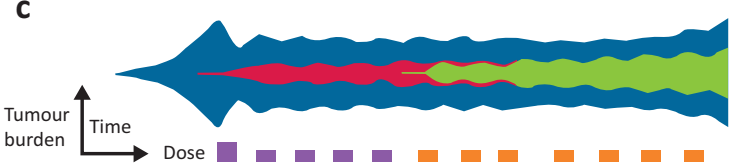

d

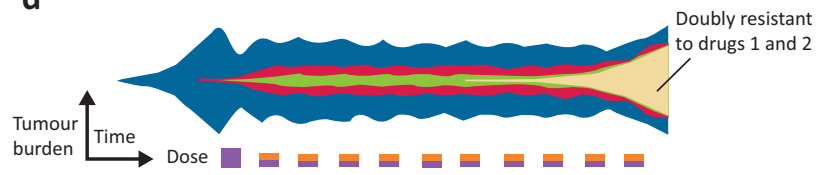

Fig 3. Cancer clonal evolution under therapy. a) Sequential, nonadaptive therapy. Conventional sequential therapy of two treatments selects for a clone resistant to treatment one (red) upon tumour relapse and subsequently a clone resistant to treatment two (green). b) Single drug adaptive therapy. Adaptive therapy maintains a stable tumour volume by introducing treatment holidays. Drug-sensitive clones (blue) suppress the growth of less-fit resistant clones (red). However, resistance still eventually occurs. c) Two drug sequential adaptive therapy. One proposed adaptive multidrug strategy is to alternately switch between drugs during each on-off cycle of tumour burden. d) Two drug concomitant adaptive therapy. An alternative multidrug adaptive strategy is to administer both drugs simultaneously during each on-off cycle, leading to a doubly resistant clone (yellow). Adapted with permission from West ], You L, Zhang ] et al. Towards multidrug adaptive therapy. Cancer Res 2020;80:1578-89.

Other examples abound: understanding diseases of the eye has benefited from imaging, laser physics, sensor technology and image analysis, optical coherence tomography is one such example that has been rapidly adopted in clinical practice to good effect in ophthalmology. ${ }^{34}$ The use of a novel near-infrared spectral imaging technique, developed in a physics laboratory, shows promise in the early detection of dental caries. ${ }^{35}$

There are, of course, many other examples of clinical translation from the physical sciences into the clinic but one particular field is perhaps rather wider in scope: computational medicine. Computational medicine applies methods from computational sciences, engineering and mathematics and to improve our understanding and treatment of human diseases. ${ }^{1,36,37}$ Could it reduce the scale and duration of expensive conventional trials? This entirely new paradigm can reduce, refine or replace conventional trials by developing in silico trials, which perform entirely, or in part, in patient-like computer simulations modelling aspects of medical device, drug effect or clinical intervention. ${ }^{38}$ Our recent work has shown how these approaches replicate findings of conventional trials and provide additional insights not accessible through conventional trials without posing risks to human or impacting animal welfare (manuscript in preparation). 
The value of interdisciplinary collaborations is not limited to biomedical research. The long-established discipline of operational research is increasingly recognised as a robust approach to designing clinical services in hospitals and entails significant interdisciplinary collaborations. ${ }^{39}$ We might anticipate wider use of this approach in, for example, determining the scale and location of hospitals in future, drawing on the work of major programmes such as the Infrastructure Transitions Research Consortium (ITRC), a consortium of seven UK universities led by the University of Oxford, which has drawn together a wide range of disciplines and created a globally unique set of simulation models and methodologies which enable long-term cross-sectoral planning of sustainable and resilient infrastructure systems. ${ }^{40}$

\section{Clinicians for future healthcare}

If interdisciplinary research is driving clinical advances, what are the consequences for clinical training, particularly given the burgeoning demands made on the curricula for all clinicians including doctors, nurses, clinical scientists and allied health professions? The principle of the 'T-shaped' researcher alluded to earlier may be helpful here: clinical skills must indeed remain core to the training programmes and, for doctors in particular, it would be unwise to freight the curriculum with advanced training in areas such as nanoscience and AI. However, one trend that is already, and rightly, evident for all professions is increased emphasis on data science and quantitative techniques, particularly as the volume of useful data increases. ${ }^{41}$ Key to making progress is the appreciation by all clinical staff of the role of interdisciplinary research and that a strong research culture comes with positive patient, staff and organisational benefits. ${ }^{20,42}$ As indicated earlier, some of the most compelling examples of progress being made are where a clinician has a 'hunch' that an answer to a particular clinical question may already exist, at least in part, in another area of science. The role of clinical scientists in hospitals (in both laboratory services and medical physics) is key here. These professions are well-placed to play a brokering role in bridging the gap between the basic sciences and clinical practice.

All this may give the impression that the professional composition of the healthcare workforce will remain unchanged; this is not likely for many reasons, but here it is worth noting that as interdisciplinary research continues to develop, new 'professions' will emerge, might we see the emergence of 'clinical mathematicians' or 'computational doctors', for example? The Moffitt Cancer Centre suggests this might occur.

\section{'Blue sky’}

Many would agree that predicting the future is fraught with difficulty. Still, in the spirit of the Future Healthcare Journal, it may be worth noting one particular development likely to have a significant impact, perhaps in the not-so-distant future; the rapidly increasing power of computing that is being driven by developments in basic science, including quantum computing. ${ }^{43}$

Quantum computing is another example of how a theory (quantum theory) can ultimately lead to very significant translational impact across a wide range of disciplines: in this case, a genuinely immense increase in computing speed. This increase in computing speed, combined with the widening range of informative clinical measurements in real-time derived from wearable technology, as well as genomic, proteomic, metabolomic, imaging, behavioural and socio-economic data, is likely to make possible a full-scale simulation of a patient trajectory and life course. ${ }^{44}$ In effect, the patient will have an avatar or digital twin who will never be late for an appointment and on whom it will be relatively straightforward to evaluate the impact of different treatments using in silico modelling.$^{45}$ This deeply interdisciplinary approach will profoundly impact the way clinical research is conducted and health services are delivered.

\section{Conclusion}

It is widely recognised that healthcare systems will face unprecedented demands driven by changing demographics, while at the same time being reshaped by an increasing emphasis on provision of care in community settings. The integration of scientific and technological breakthroughs with new and emerging care pathways will bring exceptional opportunities to improve clinical outcomes, though attention must also be paid to the attendant risk of deepening health inequalities. In a sense, interdisciplinary research is simply what is needed to make progress in addressing these long-standing and significant challenges; a hallmark of a serious attempt to answer the difficult questions in healthcare. Of course, advances made by interdisciplinary research will raise challenges (including ethical dilemmas) though interdisciplinary approaches may be better suited to addressing some of the difficult ethical problems that will arise. While the role of clinicians will continue to evolve, they will remain vital members of a much broader and more diverse interdisciplinary team, with exciting opportunities to engage with a wide range of different disciplines and playing leading roles in the healthcare of the future.

\section{Acknowledgements}

We also acknowledge useful discussions with Professors Stephen Evans and David Jayne, and Vee Mapunde (University of Leeds) and Professor John Girkin (Durham University).

\section{Conflicts of interest}

Stephen Smye is a professor in the School of Medicine at the University of Leeds and specialty cluster lead with the NIHR Clinical Research Network (until April 2021) based at King's College London. He receives funding from the UKRI-funded Physics of Life Network (standard grant EP/T022000/1) and from the Rosetrees Trust 'Physics of Medicine; building a network at the interface between medicine and the physical sciences' (grant PGS192/10092). This support is gratefully acknowledged.

Alejandro Frangi is diamond jubilee chair in computational medicine and Royal Academy of Engineering chair in emerging technologies at the University of Leeds. He acknowledges support from the Royal Academy of Engineering under a RAEng chair in emerging technologies (CiET1919/19). EPSRC partially supports his interdisciplinary work through grants TUSCA (EP/V04799X/1) and MedIAN Network (EP/N026993/1) and Cancer Research UK through the Cancer Research UK Radiation Research Centre of Excellence at the University of Leeds (C19942/A28832).

\section{References}

1 Smye SW. The physics of physik. J R Coll Physicians Edinb 2018;48: 3-8. 
2 Wilson J. Theoretical physics to pandemic modelling: Neil Ferguson on his life in science. Imperial College London, 2020. www.imperial. ac.uk/news/205053/theoretical-physics-pandemic-modelling-neilferguson [Accessed 3 February 2021].

3 Fisher CK, Walsh J. Faster, more efficient trials: novel trial designs using digital twins. Endpoints Webinars. https://webinars.endpts. com/faster-more-efficient-trials-novel-trial-designs-using-digitaltwins [Accessed 3 February2021].

4 White FM, Gatenby RA, Fischbach C. The physics of cancer. Cancer Research 2019;79:2107-10.

5 Turing AM. Computing, Machinery and Intelligence. Mind 1950;59: 433-60.

6 Maxwell PH (chair). The importance of engineering and physical sciences research to health and life sciences. Swindon: Engineering and Physical Sciences Research Council, 2014.

7 Arts and Humanities Research Council. Arts and health. AHRC. https://ahrc.ukri.org/innovation/health-and-wellbeing-researchportfolio/arts-and-health/[Accessed 4 February 2021].

8 Van Noorden R. Interdisciplinary research by the numbers. Nature 2015;525:306-7.

9 Rylance R. Grant giving: Global funders to focus on interdisciplinarity. Nature 2015;525:313-5.

10 Wu L, Wang D, Evans JA. Large teams develop and small teams disrupt science and technology. Nature 2019;566:378-82.

11 UK Research and Innovation. Interdisciplinary research. UKRI. https://re.ukri.org/research/interdisciplinary-research [Accessed 4 February 2021].

12 Brown RR, Deletic A, Wong THF. How to catalyse collaboration. Nature 2015;525:315-7.

13 Ridley A (chair). Improving recognition of team science contributions in biomedical research careers. London: Academy of Medical Sciences, 2016.

14 Mullard A. Addressing cancer's grand challenges. Nat Rev Drug Discov 2020;19:825-6.

15 Council for Science and Technology. Principles for science and technology moon-shots. Council for Science and Technology, 2020. www.gov.uk/government/publications/principles-for-science-andtechnology-moon-shots [Accessed 4 February 2021].

16 Lechler R (chair). Transforming health through innovation: Integrating the NHS and academia. London: The Academy of Medical Sciences, 2020.

17 Royal College of Physicians. Research for all: Developing, delivering and driving better research: London: RCP, 2020.

18 Surgical MedTech Co-operative. Home. Surgical MedTech Co-operative. https://surgicalmic.nihr.ac.uk [Accessed 5 February 2021].

19 University of Leeds. Bragg Centre for Materials Research. University of Leeds.www.leeds.ac.uk/info/130565/bragg_centre_for_ materials_research

20 Collins B. Adoption and spread of innovation in the NHS. London: The King's Fund, 2018.

21 Harding K, Lynch L, Porter J, Taylor NF. Organisational benefits of a strong research culture in a health service: a systematic review. Aust Health Rev 2017;41:45-53.

22 Physics of Life. Physics of Life: Welcome. Physics of Life. www.physicsoflife.org.uk [Accessed 5 February 2021].

23 UK Research and Innovation. Develop your research career. UKRI, 2021. www.ukri.org/our-work/developing-people-and-skills/ develop-your-research-career/find-fellowships-and-other-fundingfor-researchers [Accessed 5 February 2021].

24 National Institute for Health Research. NIHR Fellowship Programme. NIHR. www.nihr.ac.uk/explore-nihr/academy-programmes/fellowshipprogramme.htm [Accessed 5 February 2021].

25 UK Research and Innovation. Healthcare technologies new challenges NetworkPlus. UKRI. www.ukri.org/opportunity/healthcaretechnologies-new-challenges-networkplus [Accessed 5 February 2021].
26 University of Exeter. EPSRC hub for quantitative modelling in healthcare. University of Exeter. www.exeter.ac.uk/quantitativemodelling [Accessed 9 March 2021].

27 Cancer Research UK. Multidisciplinary Project Award. Cancer Research UK. www.cancerresearchuk.org/funding-for-researchers/ our-funding-schemes/multidisciplinary-project-award [Accessed 4 February2021].

28 National Cancer Institute. Physical Sciences in Oncology. National Cancer Institute. https://physics.cancer.gov [Accessed 5 February 2021].

29 Moffitt Cancer Center. Cancer Biology and Evolution. Moffitt Cancer Center. https://moffitt.org/research-science/research-programs/ cancer-biology-and-evolution [Accessed 5 February 2021].

30 West ], You L, Zhang ] et al. Towards multidrug adaptive therapy. Cancer Res 2020;80:1578-89.

31 Zhang J, Cunningham J], Brown JS, Gatenby RA. Integrating evolutionary dynamics into treatment of metastatic castrate-resistant prostate cancer. Nat Commun 2017;8:1816.

$32 \mathrm{~N}$ Ingram, McVeigh LE, Abou-Saleh RH et al. Ultrasound-triggered therapeutic microbubbles enhance the efficacy of cytotoxic drugs by increasing circulation and tumor drug accumulation and limiting bioavailability and toxicity in normal tissues. Theranostics 2020;10:10973-92.

33 Camm JA, Henderson R, Brisinda D et al. Clinical utility of magnetocardiography in cardiology for the detection of myocardial ischemia Journal of Electrocardiology 2019;57:10-7.

34 Adhi M, Duker JS. Optical coherence tomography - current and future applications. Current Opinion in Ophthalmology 2013;24: 213-21.

35 Bounds AD, Girkin JM. Early stage dental caries detection using near infrared spatial frequency domain imaging. Scientific Reports 2021;11:2433

36 Winslow RL, Trayanova N, Geman D, Miller MI. Computational medicine: translating models to clinical care. Sci Transl Med 2012; 4:158rv11.

37 Physiome Project. About the IUPS Physiome Project. IUPS. http:// physiomeproject.org/about/molecules-to-humankind [Accessed 5 February 2021).

38 Pappalardo F, Russo G, Tshinanu FM, Viceconti M. In silico clinical trials: concepts and early adoptions. Brief Bioinform 2019;20: 1699-708

39 MASHnet. The UK network for modelling \& simulation in healthcare. MASHnet. http://mashnet.info [Accessed 5 February 2021).

40 Infrastructure Transitions Research Consortium. Simulating the future of national infrastructure. ITRC. www.itrc.org.uk [Accessed 5 February2021].

41 West GB. The importance of quantitative systemic thinking in medicine. Lancet 2012:379;1551-9.

42 Boaz A, Hanney S, Jones T, Soper B. Does the engagement of clinicians and organisations in research improve healthcare performance: a three-stage review. BMJ Open 2015;5:e009415.

43 Kaye P, Laflamme R, Mosca M. An introduction to quantum computing. Oxford: Oxford University Press, 2007.

44 Brown S-A. Principles for developing patient avatars in precision and systems medicine. Front Genet 2015;6:365.

45 The Medical Futurist. Digital twins and the promise of personalized medicine. TMF, 2020. https://medicalfuturist.com/digital-twin-andthe-promise-of-personalized-medicine [Accessed 5 February 2021].

Address for correspondence: Prof Stephen Smye, University of Leeds School of Medicine, Worsley Building, Leeds LS2 9JT, UK. Email: s.w.smye@leeds.ac.uk 